\title{
A form of bulk silicon consisting of "magic" clusters
}

\author{
M. Grass, D. Fischer, ${ }^{a)}$ M. Mathes, G. Ganteför, P. Nielaba ${ }^{\text {b) }}$ \\ Department of Physics, University of Konstanz, D-78457 Konstanz, Germany
}

\begin{abstract}
$\mathrm{Si}_{4}$ clusters are soft-landed on an inert Van-der-Waals surface and the island formation is studied using x-ray and ultraviolet photoelectron spectroscopy. The experimental data indicate that the clusters do not fuse to $\mathrm{Si}$ islands but survive as individuals. The potential-energy surface of two $\mathrm{Si}$ clusters is calculated and a barrier against fusion is found, in agreement with the experiments. Both finding support the existence of a form of silicon consisting of $\mathrm{Si}_{4}$ clusters.
\end{abstract}

Since the discovery of the "supermagic" cluster $\mathrm{C}_{60}{ }^{1}$ the possibility of the synthesis of new materials consisting of stable clusters fascinates researchers. In case of $\mathrm{C}_{60}$ and similar fullerenes like $\mathrm{La} @ \mathrm{C}_{82}$, such materials exist and fullerite - the bulk material formed by weakly interacting $\mathrm{C}_{60}$ "soccer balls"- represents a new form of carbon. ${ }^{2}$

The question arises, whether magic clusters of other elements like $\mathrm{Si}$ or $\mathrm{Al}$ might be suitable as building blocks of cluster materials. However, many of these clusters are more reactive compared to the fullerenes, and the chemical methods ${ }^{2}$ used for the separation of fullerene materials cannot be used for clusters of most other elements. In experimental studies of free clusters many other stable clusters have been found, such as $\mathrm{C}_{32}{ }^{3}$ or $\mathrm{Si}_{4} \cdot{ }^{4,5}$ In addition, there are predictions of possible building blocks of new materials like $\mathrm{Al}_{13} \mathrm{H}^{6,7}$ and $\mathrm{Si}_{45}{ }^{8}$

For the studies published so far, relative stability is the only criterion making a cluster a candidate as a building block for new material. However, even more important is the interaction between neighboring clusters. In cluster materials neighboring clusters "touch" each other, and there must be a barrier against fusion.

Here we present combined experimental and theoretical results on the interaction between neighboring magic clusters. As a firs example, magic $\mathrm{Si}_{4}$ clusters are selected. Experimentally, the clusters are mass-selected and soft landed on an inert surface. ${ }^{9}$ They are highly mobile on this surface at room temperature and will form islands of bulk $\mathrm{Si}$ if there is no barrier. ${ }^{10-12}$ The samples are studied using X-ray and ultraviolet photoelectron spectroscopy (XPS, UPS) and, in contrast to an earlier study of $\mathrm{Si}_{10}$ on amorphous carbon, ${ }^{13}$ the spectra contradict the formation of islands, supporting the existence of a barrier. The interaction potential between two approaching $\mathrm{Si}_{4}$ clusters is calculated for two geometries, and for both channels a barrier is found. Accordingly, a new bulk consisting of $\mathrm{Si}_{4}$ clusters should exist.

Density functional calculations for the $\mathrm{Si}_{4}$ clusters have been performed with the gradient-corrected exchangecorrelation (xc) functionals of Perdew, Burke and Ernzerhof

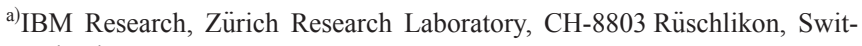
zerland.

${ }^{b)}$ Electronic mail: peter.nielaba@uni-konstanz.de
(PBE) ${ }^{14}$ The computational details are similar to the studies of $\mathrm{Au},{ }^{15-19}$ modifie to the case of $\mathrm{Si}^{20}$

In a firs step, the ground state structure of a $\mathrm{Si}_{4}$ cluster was determined. The $\mathrm{Si}_{4}$ cluster forms a planar rhombohedric structure with two sharp and two fla corners. Fixing the distance $R$ between two $\mathrm{Si}$ atomic centers on the $x$-axis in two different $\mathrm{Si}_{4}$ clusters, the potential energy surface was
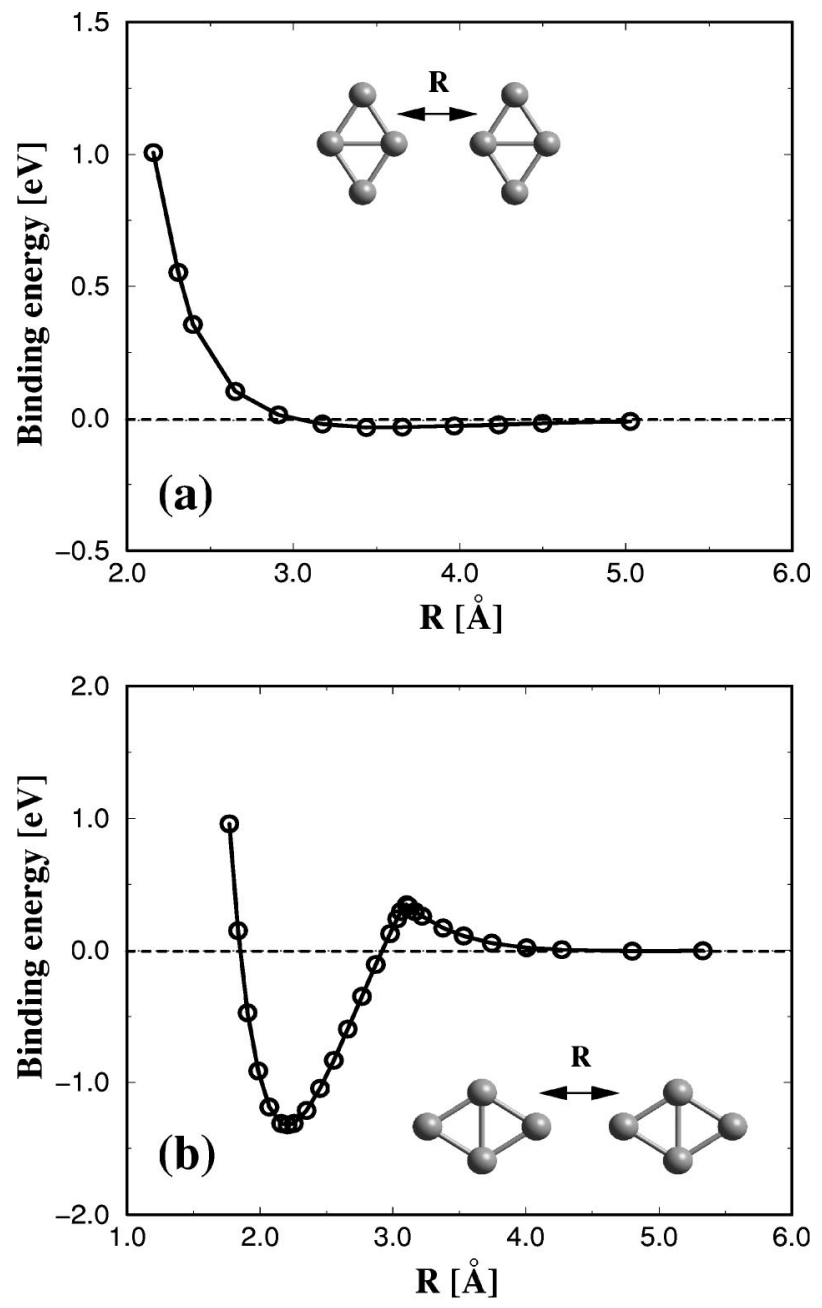

FIG. 1. Calculated potential energy curves for two neutral interacting $\mathrm{Si}_{4}$ clusters. Two different reaction channels have been calculated: the two tetramers approaching each other with the fla (a) and sharp (b) corners ahead. Case (a) is repulsive, while in case (b) a bond is formed. In (b) an energy barrier is observed at a distance of $3.1 \mathrm{~A}$ and a height of $0.3 \mathrm{eV}$. 


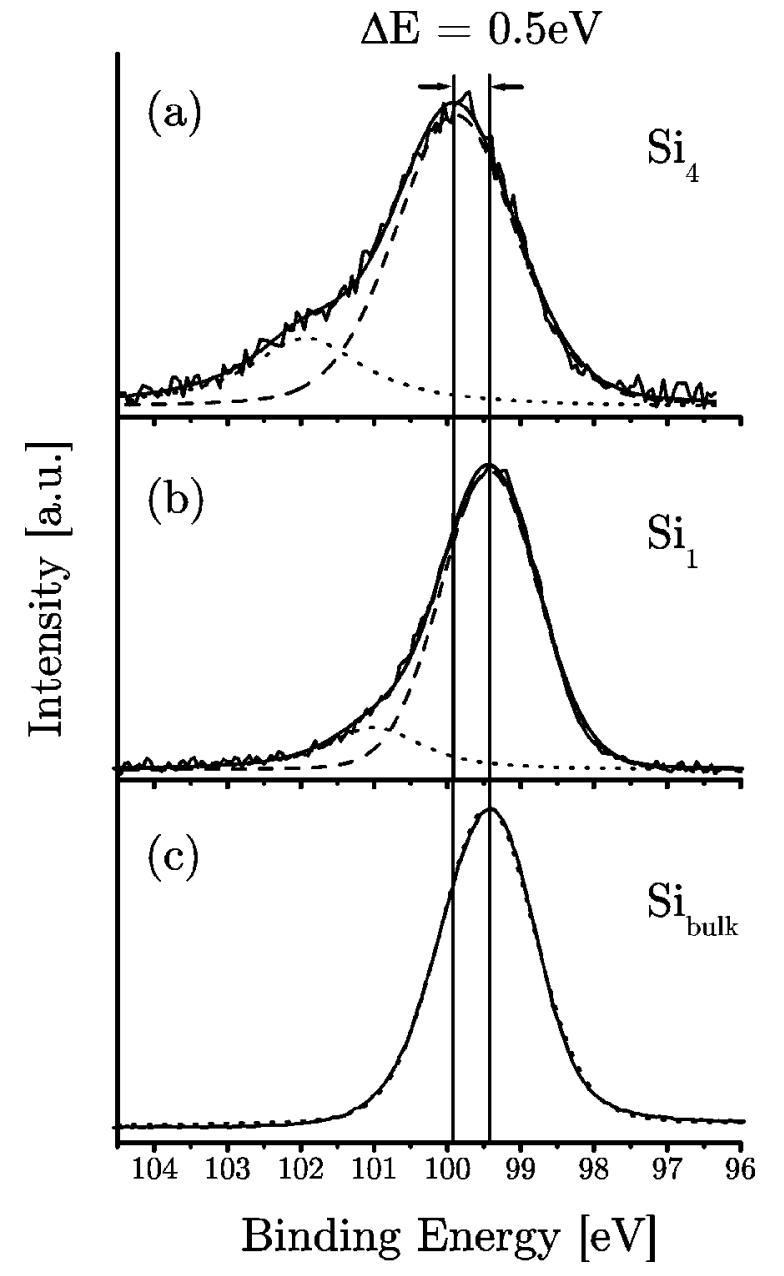

FIG. 2. XPS spectra of the $\mathrm{Si} 2 \mathrm{p}$ peak recorded for (a) $\mathrm{Si}_{4}$ clusters deposited at $5 \mathrm{eV}$ onto HOPG ( $2 \%$ coverage), (b) positively charged $\mathrm{Si}$ atoms deposited at $5 \mathrm{eV}$ onto HOPG (2\% coverage) and (c) for a Si bulk reference sample. Fits of the experimental data are also displayed.

calculated. Two different "reaction channels" have been considered (see Fig. 1) starting with structures with symmetry about the $x$-axis. In Fig. 1(a) the distance between the two $\mathrm{Si}$ atoms at the fla angles is fixed and in Fig. 1(b) the distance of the atoms at the sharp angles is fixed The total energy is displayed in Fig. 1 as a function of $R$.

The experimental setup has been described in detail elsewhere. ${ }^{9}$ In short, cluster ions are generated using a cluster ion source and mass separated. The ions are decelerated down to a kinetic energy of $1.2 \mathrm{eV} /$ atom and landed on highly oriented pyrolytic graphite (HOPG). The sticking coefficien is only about $10 \%,{ }^{21}$ and a coverage of $2 \%$ of $\mathrm{Si}$ atom monolayer (ML) has been achieved after $2 \mathrm{~h}$. There is no generation of surface defects at this low kinetic energy. ${ }^{9-12}$ We can not exclude cluster fragmentation, but it seems unlikely at this low kinetic energy compared to the binding energy of the clusters. ${ }^{9,12}$ The clusters on the surfaces are studied using XPS and UPS in situ. . $^{911,22,23}$

Figure 1 displays the calculated dependencies of potential energy of two interacting $\mathrm{Si}_{4}$ as a function of distance. There are several geometries possible for two tetramers approaching each other. We assume the $\mathrm{Si}_{4}$ clusters lie fla on the surface and, therefore, we are restricted to planar geometry of two $\mathrm{Si}_{4}$ approaching each other with the two obtuse (a) or sharp (b) corners encountering one another. For the

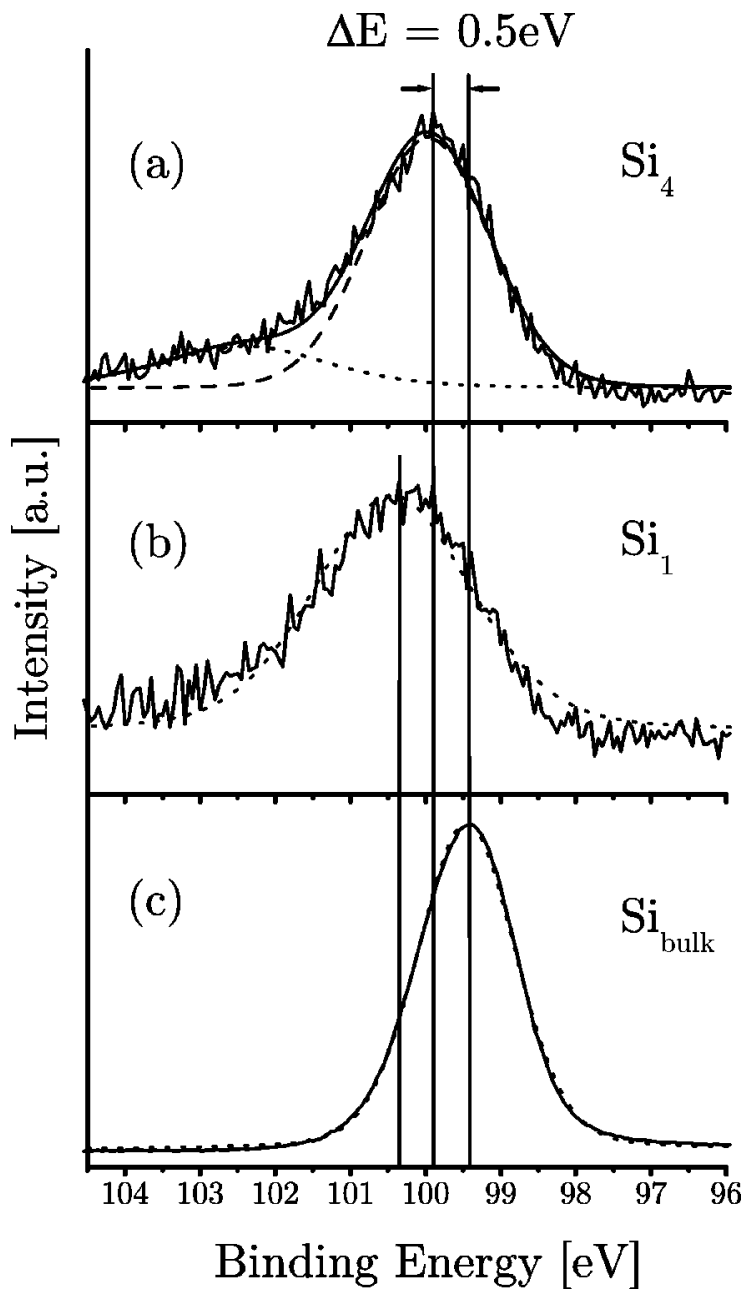

FIG. 3. XPS spectra of the $\mathrm{Si} 2 p$ feature recorded for (a) $\mathrm{Si}_{4}$ clusters deposited at $5 \mathrm{eV}$ onto amorphous carbon ( $4 \%$ coverage), (b) positively charged $\mathrm{Si}$ atoms deposited at $5 \mathrm{eV}$ onto amorphous carbon ( $3 \%$ coverage) and (c) for a Si bulk reverence sample.

geometry displayed in Fig. 1(a) the potential energy corresponds to a repulsive interaction. If the two $\mathrm{Si}_{4}$ approach with the sharp corners ahead a bond is formed [Fig. 1(b)] with a binding energy of $1.3 \mathrm{eV}$.

Important in Fig. 1(b) is the small increase of the potential energy at a distance of $3.1 \mathrm{~A}$. This barrier is $0.3 \mathrm{eV}$ high and, therefore, it might not be overcome at kinetic energies corresponding to room temperature. Accordingly, for both channels a repulsive interaction at low temperatures is predicted.

These theoretical finding support the results of an experimental study of $\mathrm{Si}_{4}$ clusters deposited on HOPG at room temperature. We assume that the interaction with the substrate is weak and no change of the structure of the $\mathrm{Si}_{4}$ is to be expected. The clusters most likely lie fla on the surface, are highly mobile at room temperature and approach each other in geometries similar to the ones calculated. The samples are studied using XPS and only the peak corresponding to the $2 p$ core level of $\mathrm{Si}$ is examined (Fig. 2). For clean bulk $\mathrm{Si}$ an almost symmetric peak centered at a binding energy of $99.4 \mathrm{eV}$ is observed [Fig. 2(c)]. For Si atom ions on HOPG [Fig. 2(b)] a similar peak is observed with a shoulder corresponding to a feature at $101 \mathrm{eV}$ binding energy. For $\mathrm{Si}_{4}$ clusters on HOPG [Fig. 2(a)] a shift of the main peak of 


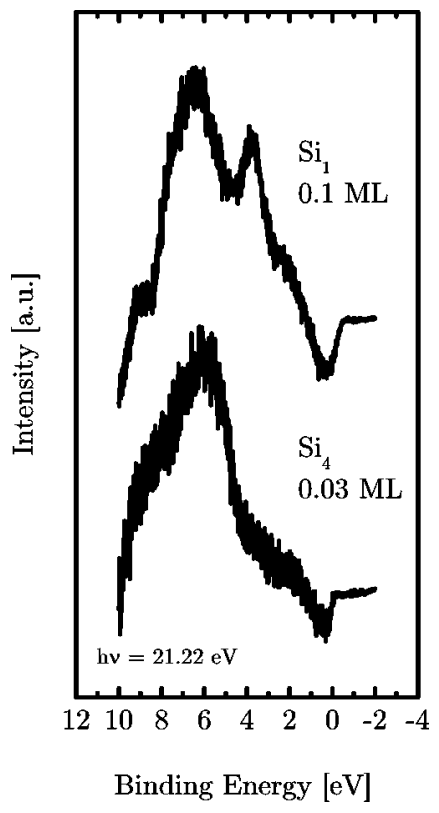

FIG. 4. UPS $(\mathrm{h} \nu=21.22 \mathrm{eV})$ data of deposited $\mathrm{Si}_{1}^{+}$ions $(10 \%$ coverage $)$ and $\mathrm{Si}_{4}^{-}$cluster anions $(3 \%$ coverage $)$ on amorphous carbon at room temperature. The data have been obtained by subtracting the spectrum of the clean carbon substrate from the spectrum of the substrate with the $\mathrm{Si}$ atoms or clusters deposited.

$0.5 \mathrm{eV}$ towards higher binding energy and a shoulder corresponding to a peak around $102 \mathrm{eV}$ is observed.

The small features at binding energies around 101-102 $\mathrm{eV}$ are assigned to Si bound to carbon. The main peak corresponds to Si bound to Si. The shift of $0.5 \mathrm{eV}$ between the deposited clusters and the deposited atoms is essential. We explain this shift by a lower average coordination number of the Si atoms in clusters. Similar shifts have been observed in XPS spectra of various mass-selected deposited clusters. $^{11,13,22,23}$ In these experiments the clusters are not mobile on the surface ${ }^{13,22}$ or, if the clusters can diffuse, the shift vanishes with time due to the formation of bulk-like islands. ${ }^{11}$ In our case the clusters are mobile, ${ }^{9,11}$ but the shift does not vanish with time. In case of the deposited atoms there is no shift corresponding to the formation of bulk-like islands. These finding indicate that deposited $\mathrm{Si}_{4}$ clusters do not form larger islands, but remain as individual clusters on the surface.

To support our findings we repeated the above experiments with amorphous carbon. This surface has a high number of dangling carbon bonds. Figure 3 displays the XPS data. For the deposition of Si atom ions [Fig. 3(b)], almost no pure $\mathrm{Si}-\mathrm{Si}$ bonds, but only $\mathrm{Si}$ atoms bound to carbon are found. No Si islands are formed, but the Si atoms are trapped at surface defects.

In contrast, the XPS feature corresponding to the soft landed $\mathrm{Si}_{4}$ cluster ions [Fig. 3(a)] does not exhibit a difference to the case of the HOPG surface [Fig. 2(a)]. The tetramers do not react with defect sites of the amorphous carbon and do not coalesce.

For further support we studied the samples with UPS. At the photon energy used here $(h \nu=21.22 \mathrm{eV})$ the cross section for photoemission from silicon is rather low and we did not succeed in obtaining UPS data for HOPG substrates. Figure 4 displays a comparison of UPS data of $\mathrm{Si}$ atoms and
$\mathrm{Si}_{4}$ clusters on amorphous carbon. The data correspond to the difference between the UPS spectra of the sample with clusters and the clean sample. In both spectra a broad peak at 6-7 eV binding energy is observed, which can be assigned to Si-derived valence orbitals. For the deposited atoms, an additional peak at $4 \mathrm{eV}$ is observed, which we tentatively assign to the formation of $\mathrm{Si}-\mathrm{C}$, in agreement with the XPS data (Fig. 3). The $\mathrm{Si}_{4}$ sample does not exhibit such a feature. Both the XPS and UPS data for amorphous carbon show that the $\mathrm{Si}_{4}$ clusters are rather inert.

In conclusion, we studied the interaction of neighboring $\mathrm{Si}_{4}$ clusters theoretically and experimentally. Both sets of data support the idea that this magic silicon cluster is suitable as a building block for a new cluster material. In the calculations, a repulsive barrier has been found in the interaction potential that blocks spontaneous fusion of clusters at suffi ciently low temperature. Experimentally, for clusters deposited on an inert surface at room temperature, no tendency to form larger islands of bulk $\mathrm{Si}$ is observed.

We gratefully acknowledge useful discussions with W. Andreoni and A. Curioni. Support by the SFB 513 and computer time from the SSC Karlsruhe is gratefully acknowledged.

${ }^{1}$ H. W. Kroto, J. R. Heath, S. C. O’Brien, R. F. Curl, and R. E. Smalley, Nature (London) 318, 162 (1985).

${ }^{2}$ M. S. Dresselhaus, G. Dresselhaus, and P. C. Eklund, Science of Fullerenes and Carbon Nanotubes (Academic, San Diego, 1995).

${ }^{3}$ H. Kietzmann, R. Rochow, G. Ganteför, W. Eberhardt, K. Vietze, G. Seifert, and P. W. Fowler, Phys. Rev. Lett. 81, 5378 (1998).

${ }^{4}$ O. Cheshnovsky, S. H. Yang, C. L. Pettiette, M. J. Craycraft, Y. Liu, and R. E. Smalley, Chem. Phys. Lett. 138, 119 (1987).

${ }^{5}$ J. Müller, Bei Liu, A. A. Shvartsburg, S. Ogut, J. R. Chelikowsky, K. W. M. Siu, Kai-Ming Ho, and G. Ganteför, Phys. Rev. Lett. 85, 1666 (2000).

${ }^{6}$ S. N. Khanna and P. Jena, Chem. Phys. Lett. 218, 383 (1993).

${ }^{7}$ S. Burkart, N. Blessing, B. Klipp, J. Müller, G. Ganteför, and G. Seifert, Chem. Phys. Lett. 301, 546 (1999).

${ }^{8}$ U. Röthlisberger, W. Andreoni, and M. Parrinello, Phys. Rev. Lett. 72, 665 (1994).

${ }^{9}$ B. Klipp, M. Grass, J. Müller, D. Stolcic, U. Lutz, G. Ganteför, T. Schlenker, J. Boneberg, and P. Leiderer, Appl. Phys. A: Mater. Sci. Process. 73, 547 (2001).

${ }^{10}$ P. Scheier, B. Maersen, M. Lonfat, W.-D. Schneider, and K. Sattler, Surf. Sci. 458, 113 (2000).

${ }^{11}$ W. Yamaguchi, K. Yoshimura, Y. Maruyama, K. Igaraschi, S. Tanemura, and J. Murakami, Chem. Phys. Lett. 311, 415 (1999).

${ }^{12}$ S. J. Caroll, S. G. Hall, R. E. Palmer, and R. Smith, Phys. Rev. Lett. 81, 3715 (1998).

${ }^{13}$ J. E. Bower and M. Jarrold, J. Chem. Phys. 97, 8312 (1992).

${ }^{14}$ J. P. Perdew, K. Burke, and M. Ernzerhof, Phys. Rev. Lett. 77, 3865 (1996).

${ }^{15}$ H. Grönbeck and W. Andreoni, Chem. Phys. 262, 1 (2000).

${ }^{16}$ H. Grönbeck, A. Curioni, and W. Andreoni, J. Am. Chem. Soc. 122, 3839 (2000); W. Andreoni, A. Curioni, and H. Grönbeck, Int. J. Quantum Chem. 80, 598 (2000).

${ }^{17}$ D. Fischer, W. Andreoni, A. Curioni, H. Grönbeck, S. Burkart, and G. Ganteför, Chem. Phys. Lett. 361, 389 (2002).

${ }^{18}$ R. Car and M. Parrinello, Phys. Rev. Lett. 55, 2471 (1985).

${ }^{19}$ Calculations used the CPMD code by J. Hutter: CPMD 3.0, copyright IBM Corporation (1990-1997) and MPI Festkörperforschung Stuttgart, 1997.

${ }^{20} \mathrm{Si}$ PBE pseudopotentials and cluster structures from W. Andreoni (unpublished).

${ }^{21}$ W. Yamaguchi, K. Yoshimura, Y. Tai, Y. Maruyama, K. Igarashi, S. Tanemura, and J. Murakami, J. Chem. Phys. 112, 9961 (2000).

${ }^{22}$ W. Eberhardt, P. Fayet, D. M. Cox, Z. Fu, A. Kaldor, R. Sherwood, and D. Sondericker, Phys. Rev. Lett. 64, 780 (1990).

${ }^{23}$ G. K. Wertheim and S. B. DiCenzo, Phys. Rev. B 37, 844 (1988). 